\title{
The status of the C 3 and C4 fractions of the complement system in children with Henoch-Schönlein purpura
}

\author{
Khrystyna Chaika, Nataliia Makieieva, Oksana Afanasieva, Mariia Yavorovych \\ $2^{\text {nd }}$ Department of Pediatrics, Kharkiv National Medical University, Kharkiv, Ukraine
}

\section{ABSTRACT}

\begin{abstract}
Aim of the study: To determine the interaction of components of complement C3 and C4 with indicators of unspecific immune system and biological inflammatory markers.

Material and methods: A total of 80 children were recruited, including 60 patients with Henoch-Schönlein purpura (HSP) aged from 2 to 17 years old and 20 healthy children of the same age. The HSP patients were divided into two groups: an HSPWN group $(n=47)$, including HSP patients without renal involvement; and an HSPN group $(n=13)$, including patients with HSP combined with renal syndrome. The control group comprised 20 healthy children. Standard tests, clinical examination, and measurement of the levels of C3 and C4 complement fractions were performed on admission and during remission time. The components of the complement system C3 and C4 were determined by ELISA using standard ELISA C3 and C4 kits from AssayPro, USA.

Results: It was significantly proved the difference between medians in all groups due to high criteria $\mathrm{H}$ for C3 in acute and remission periods $(H=15.58, p=0.000$ and $H=14.58, p=0.00$, respectively). The serum level of C3 was significantly decreased in patients of HSPWN and HSPN groups in the acute period $\left(p_{\mathrm{HSPWN}-\mathrm{C}}=0.000\right.$, $\left.p_{\mathrm{HSPN}-\mathrm{C}}=0.004\right)$ and during remission $\left(p_{\mathrm{HSPWN}-\mathrm{C}}=0.000, p_{\mathrm{HSPN}-\mathrm{C}}=0.018\right)$, compared with controls. The serum level of $\mathrm{C} 4$ was significantly decreased in patients in the HSPWN group in the acute period compared with patients on the HSPN group $\left(p_{\text {HSPWN-HSPN }}=0.020\right)$. The level of C4 was significantly lower during remission time in both groups $(T=144.0, p=0.041$ and $T=10.5, p=0.025$, respectively).

Conclusions: Abnormalities of the complement system in HSP have been reported. Hypocomplementaemia was not dependent on the severity of the process of HSP. In the period of remission, the levels of C3 and C4 approached the control group's values.
\end{abstract}

KEY WORDS:

child, C3, C4, Henoch-Schönlein purpura.

\section{INTRODUCTION}

Haemorrhagic vasculitis, or Henoch-Schönlein purpura (HSP), is an immunopathological disease characterised by systemic inflammation of small vessels with immune deposition in their walls, mainly consisting of class A immunoglobulins (IgA), which are manifested in symmetric, often small-scale haemorrhages, usually in combination with damage to the joints, gastrointestinal tract, and kidneys [1]. More than $75 \%$ of children with HSP are under 10 years old, and the peak incidence is between four and six years old [2]. The prevalence of HSP ranges from 10 to 20 cases per 100,000 children under the age of 17 years [3]. HSP nephritis occurs in $20-50 \%$

\section{ADDRESS FOR CORRESPONDENCE:}

Khrystyna Chaika, $2^{\text {nd }}$ Department of Pediatrics, Kharkiv National Medical University, 2 Pasteur St., 61075 Kharkiv, Ukraine, ORCID: 0000-0002-6756-6469, e-mail: potikhenska@gmail.com 
of children and can lead to complications [4]. Some biomarkers of HSP have been studied, which showed development of renal complications such as serum Gd-IgA1 and urinary $\operatorname{Ig} \mathrm{A}, \operatorname{IgG}$, IgM, IL-8, IL-10, and IgA-IgG and IgA-sCD89 complexes [4], (S)-3-hydroxyisobutyric acid, p-cresol sulphate, and 3-carboxy-4-methyl-5-pentyl2 -furanpropanoic acid [5], IL-6, serum amyloid A [6].

The basis of the development of HSP is the formation of immune complexes and activation of components of the complement system, which have a damaging effect on the vascular wall. As a result, microvessels undergo aseptic inflammation with fibrinoid necrosis, perivascular oedema, microcirculation blockage, leukocyte infiltration, thrombus formation, haemorrhages, and dystrophic changes as severe as necrosis in lesion areas. Evidence of complement activation in HSP glomerulonephritis comes from characteristic patterns of a decrease in the serum concentrations of specific components, some of which are virtually diagnostic of certain nephritides. These patterns are often accompanied by the presence of complement components in the glomeruli and the detection of complement breakdown products in the circulation. In certain diseases, circulating complement-activating substances can be detected [7]. Some studies show an increase in C3, C4, and IgA in the serum of children with HSP, which indicates the involvement of immunological components in the pathogenesis of the disease. However, there are studies that demonstrate the presence of hypocomplementaemia in children with HSP, namely a low content of C3 and/or C4 [8].

Despite the urgency of the problem of HSP, the state of the vascular wall, the immunological mechanisms of damage to the vascular endothelium, the role of the complement system, and endothelial dysfunction in this disease remain insufficiently studied. In this study, the relationship of individual components of the complement system (C3 and C4) in serum with changes in homeostasis was studied. We determine the interaction of components of complement with indicators of unspecific immune system and biological inflammatory markers.

\section{MATERIAL AND METHODS}

\section{GENERAL INFORMATION}

The study was performed between January 1st, 2015 and November 1st, 2018 at the Kharkiv City Clinical Children's Hospital No. 16. A total of 80 children were recruited, including $60 \mathrm{HSP}$ patients aged from 2 to 17 years old and 20 healthy children of the same age. The HSP patients were divided into two groups: HSPWN $(n=47)$ - HSP patients without renal involvement; and HSPN $(n=13)$ - patients with HSP combined with renal syndrome. The HSPWN group comprised 32 males and 15 females, and the HSPN group comprised seven males and six females. The control group comprised 20 healthy children (with similar age and gender) presented to Kharkiv City Outpatient Hospital No. 16 for routine health control or vaccination. The diagnosis of HSP was established according to the criteria defined by the European League Against Rheumatism and the Paediatric Rheumatology European Society [9]. The criteria for inclusion in the study were verified diagnosis of HSP and the presence of informed consent from parents and/or patients. The criteria of exclusion in the study were refusal of parents and/or patients from the investigation, patients with hereditary diseases of the blood system, and other acute or chronic inflammatory diseases.

\section{METHODS}

The patients were hospitalised within the first 72 hours from the onset of the disease. The main methods of examination were the study of complaints, life and medical histories, objective examination data, and clinical, laboratory, and instrumental data. Clinical symptoms were fixed, such as purpura, arthralgia or arthritis, and abdominal pain. Manifesting of haematuria and/or proteinuria, and nephritis during the first month of HSP was diagnosed as renal syndrome (proteinuria $>0.3 \mathrm{~g} /$ day or haematuria or red blood cell casts $>5$ red blood cells/high-power field or $\geq 2+$ on a dipstick). Standard tests included routine CBC and urine test, levels of $\mathrm{C}$-reactive protein (CRP), proteins of acute inflammation, prothrombin time, and activated partial thromboplastin time (aPTT). Clinical examination was performed on admission and in remission time (after disappearance of skin syndrome) on the $7^{\text {th }}-10^{\text {th }}$ day. The levels of $\mathrm{C} 3$ and $\mathrm{C} 4$ complement fractions were studied at admission (until the development of renal syndrome) and remission time in HSP patients, and only at baseline in the control group. The components of the complement system $\mathrm{C} 3$ and $\mathrm{C} 4$ were determined by ELISA using standard ELISA C3 and C4 kits from AssayPro, USA (EC2101-1 and EC 2102-1) [10, 11]. All serum samples were frozen at $-20^{\circ} \mathrm{C}$ (for $\mathrm{C} 3$ ) and $-60^{\circ} \mathrm{C}$ (for C4) until assays.

\section{ETHICAL APPROVAL AND CONSENT TO PARTICIPATE}

All participants and their parents were informed about the research and provided written, informed consent. The study was approved by the Ethics Committee of the Kharkiv National Medical University (Ethics Commission protocol No. 8, October 5th, 2016), Ukraine and was conducted according to the guidelines of the Helsinki Declaration (1975).

\section{STATISTICAL ANALYSES}

Statistical analyses were performed using StatSoft Statistica Version 8 (Tulsa, OK). The verification of the distribution according to the Gauss law was carried out using the Shapiro-Wilk test. Non-parametric variables were 
presented as median $(\mathrm{Me})$ and interquartile range (lower quartile - Lq; upper quartile - Uq). The Kruskal-Wallis One-Way Analysis of Variance (ANOVA) on Ranks $(H)$ was used for testing statistically significant differences in the median values among all groups. Significance point was defined using Bonferroni adjustment. While comparing groups with controls, the Bonferroni correction was calculated according to the formula $p^{\prime}=p / n-1$, where $n$ is the number of patients in the group in the experiment, $p \leq 0.025$. To compare two independent samples the non-parametric Mann-Whitney $U$-test was applied, and to compare two dependent samples the non-parametric Wilcoxon test $(T)$ was used. All $p$-values were two-tailed, and values $<0.05$ were considered statistically significant. The relationship between the series of indicators was assessed using Spearman's rank correlation methods $(r)$.

\section{RESULTS}

Sixty children aged from 1 to 17 years with HSP were under observation. The distribution of children by sex among the total number of patients did not reveal a statistically significant difference: boys - 35 (58.30\%), girls $-25(41.70 \%)$. The ratio of boys to girls was $1.4: 1$.

On admission to hospital, the complaints included: unwellness $35.01 \%$ (21/60), sleep disturbance $18.33 \%$ $(11 / 60)$, and loss of appetite $33.33 \%(20 / 60)$ - most of them were of moderate or severe state $78.33 \%$ (47/60).

All of the children with HSP had complaints of haemorrhagic rash - 100\% (60/60). Joint syndrome was fixed in $36.62 \%$ of children (22/60). Arthralgia was recorded in $68.21 \%$ of children $(15 / 22)$ and $31.83 \%(7 / 22)$ registered arthritis with oedema and restriction of movement. $13.42 \%$ of children $(3 / 22)$ had typical lesions of the elbow, $26.81 \%(6 / 22)$ the knee, and $68.21 \%$ of children $(15 / 22)$ registered the defeat of step ankle joints. Abdominal syndrome was recorded in $28.33 \%$ of children (17/60). The main symptoms were: abdominal pain - in $87.01 \%$ of children (15/17); and nausea and vomiting - in $13.01 \%$ of children $(2 / 17)$.

In $44.82 \%$ of children $(27 / 60)$ acute infectious disease preceded the development of HSP. In $11.62 \%$ of children (7/60) disease began after initiation of a diet. HSP developed after being bitten by an insect in $3.32 \%$ of children $(2 / 60)$. Triggers were not found in $13.28 \%$ of children $(8 / 60)$.

There was no significant difference between sex and age, haemoglobin, CRP, prothrombin time, and aPTT, glomerular filtration rate (GFR), systolic blood pressure (SBP), and diastolic blood pressure (DBP) (Table 1). There was a significant difference between HSPWN and HSPN when determining the levels of fibrinogen and platelets in the acute period: the level of fibrinogen was elevated in the HSPN group compared with the HSPWN group, whereas platelet count was higher in the HSPN group than in the HSPWN group.

The ANOVA test showed high criteria $\mathrm{H}$ (Table 2) for $\mathrm{C} 3$ in acute and remission periods $(H=15.58$, $p=0.000$ and $H=14.58, p=0.000$, respectively), which significantly proved the difference between medians in all groups. There were no significance differences between C4 medians in all groups during acute and remission pe-

TABLE 1. Clinical and laboratory data in children with Henoch-Schönlein purpura (HSP) in the acute period

\begin{tabular}{|l|c|c|c|}
\hline Parameter & $\begin{array}{c}\text { HSPWN } \\
n=47\end{array}$ & $\begin{array}{c}\text { HSPN } \\
n=13\end{array}$ & $p$-value \\
\hline Age, years & $6.8(3.2 ; 15.5)$ & $6.6(3.8 ; 9.8)$ & 0.431 \\
\hline Males, $n(\%)$ & $28(59.57)$ & $7(53.83)$ & 0.481 \\
\hline Females, $n(\%)$ & $19(40.43)$ & $6(46.17)$ & 0.551 \\
\hline Reb blood cell count, $\times 10^{12} / \mathrm{l}$ & $3.9(3.7 ; 4.3)$ & $4.4(4.0 ; 4.8)$ & 0.000 \\
\hline Haemoglobin, g/l & $124.8(117.2 ; 137.2)$ & $128.6(118.4 ; 132.1)$ & 0.152 \\
\hline White blood cell count, $\times 10^{9} / \mathrm{I}$ & $6.8(5.0 ; 8.2)$ & $10.9(4.1 ; 13.9)$ & 0.004 \\
\hline Platelet count, $\times 10^{9} / \mathrm{l}$ & $215(190 ; 342)$ & $194(156 ; 256)$ & 0.021 \\
\hline ESR, mm/h & $16.5(6.1 ; 20.1)$ & $19.5(10.3 ; 35.1)$ & 0.342 \\
\hline Fibrinogen, $\mathrm{mg} / \mathrm{l}$ & $3685.4(3644.0 ; 3726.8)$ & $4762(4737.3 ; 4786.7)$ & 0.001 \\
\hline CRP, mg/l & $8.99(6.9 ; 10.1)$ & $9.3(6.2 ; 11.4)$ & 0.671 \\
\hline aPTT, sec & $23.76(20.82 ; 26.66)$ & $23.68(18.83 ; 28.53)$ & 0.971 \\
\hline Prothrombin time, sec & $11.27(9.76 ; 12.78)$ & $12.12(9.8 ; 14.42)$ & 0.121 \\
\hline GFR, ml/min & $110(103 ; 125)$ & $92(82 ; 100)$ & 0.210 \\
\hline SBP, SD score & $0.50 \pm 0.70$ & $0.57 \pm 0.75$ & 0.483 \\
\hline DBP, SD score & $0.58 \pm 0.54$ & $0.59 \pm 0.57$ & 0.617 \\
\hline
\end{tabular}

HSPWN - HSP patients without renal involvement, HSPN - patients with HSP combined with renal syndrome, ESR - erythrocyte sedimentation rate, CRP - C-reactive protein, aPTT - activated partial thromboplastin time, GFR - glomerular filtration rate, SBP - systolic blood pressure, DBP - diastolic blood pressure; statistical significance $p<0.05$ 
TABLE 2. The levels of $\mathrm{C} 3$ and $\mathrm{C} 4$ complement fractions in the acute period and in remission in children from the HSPWN and HSPN groups and the control group

\begin{tabular}{|c|c|c|c|c|c|}
\hline \multicolumn{2}{|c|}{$\begin{array}{l}\text { Complement } \\
\text { level }(g / l)\end{array}$} & $\begin{array}{l}\text { HSPWN } \\
(n=31) \\
\end{array}$ & $\begin{array}{c}\text { HSPN } \\
(n=13) \\
\end{array}$ & $\begin{array}{l}\text { Control group } \\
\qquad(n=20)\end{array}$ & $p$-value \\
\hline \multicolumn{6}{|c|}{ Acute period } \\
\hline C3 & $\begin{array}{l}\mathrm{Me} \\
\mathrm{Lq} \\
\mathrm{Uq}\end{array}$ & $\begin{array}{l}1.21 \\
1.04 \\
1.36 \\
\end{array}$ & $\begin{array}{l}1.18 \\
1.04 \\
1.35 \\
\end{array}$ & $\begin{array}{l}1.45 \\
1.32 \\
1.58 \\
\end{array}$ & $\begin{array}{c}p_{\text {HSPWN-C }}=0.000175 \\
p_{\text {HSPN-C }}=0.004034 \\
p_{\text {HSPWN-HSPN }}=0.9078\end{array}$ \\
\hline C4 & $\begin{array}{l}\mathrm{Me} \\
\mathrm{Lq} \\
\mathrm{Uq}\end{array}$ & $\begin{array}{l}0.37 \\
0.32 \\
0.42 \\
\end{array}$ & $\begin{array}{l}0.39 \\
0.35 \\
0.45 \\
\end{array}$ & $\begin{array}{l}0.38 \\
0.32 \\
0.44 \\
\end{array}$ & $\begin{array}{c}p_{\text {HSPWN-C }}=0.7647 \\
p_{\text {HSPN-C }}=0.4606 \\
p_{\text {HSPWN-HSPN }}=0.02075\end{array}$ \\
\hline \multicolumn{6}{|c|}{ Remission } \\
\hline C3 & $\begin{array}{l}\mathrm{Me} \\
\mathrm{Lq} \\
\mathrm{Uq}\end{array}$ & $\begin{array}{l}1.14 \\
0.87 \\
1.37 \\
\end{array}$ & $\begin{array}{l}1.24 \\
0.93 \\
1.39 \\
\end{array}$ & $\begin{array}{l}1.45 \\
1.32 \\
1.58 \\
\end{array}$ & $\begin{array}{l}p_{\text {HSPWN-C }}=0.000197 \\
p_{\text {HSPN-C }}=0.018296 \\
p_{\text {HSPWN-HSPN }}=0.3544\end{array}$ \\
\hline C4 & $\begin{array}{l}\mathrm{Me} \\
\mathrm{Lq} \\
\mathrm{Uq}\end{array}$ & $\begin{array}{l}0.34 \\
0.28 \\
0.37\end{array}$ & $\begin{array}{l}0.35 \\
0.27 \\
0.38\end{array}$ & $\begin{array}{l}0.38 \\
0.32 \\
0.44\end{array}$ & $\begin{array}{c}p_{\text {HSPWN-C }}=0.005218 \\
p_{\text {HSPN-C }}=0.1501 \\
p_{\text {HSPWN-HSPN }}=0.9692\end{array}$ \\
\hline
\end{tabular}

HSPWN - Henoch-Schönlein purpura (HSP) patients without renal involvement, HSPN - patients with HSP combined with renal syndrome, Me - median, Lq - lower quartile, Uq - upper quartile

riods. The serum level of C3 was significantly decreased in patients in the HSPWN and HSPN groups in the acute period ( $p \mathrm{HSPWN}-\mathrm{C}=0.000, p \mathrm{HSPN}-\mathrm{C}=0.004$ $[p \leq 0.025$ according to Bonferroni adjustment]) and remission ( $p \mathrm{HSPWN}-\mathrm{C}=0.000, p \mathrm{HSPN}-\mathrm{C}=0.018$ ) compared with controls. The serum level of $\mathrm{C} 4$ was significantly decreased in patients in the HSPWN group in the acute period compared with patients on the HSPN group ( $p$ HSPWN-HSPN $=0.020$ ), and in the remission period in the HSPWN compared with controls ( $p$ HSPWN-C $=0.005$ ). Wilcoxon test showed that the level of C3 fractions did not change in its dynamics in both groups (HSPWN and HSPN) $(T=204.5, p=0.394$ and $T=44.0, p=0.916$, respectively); the level of $\mathrm{C} 4$ was significantly lower during remission time in both HSPWN and HSPN groups $(T=144.0, p=0.041$ and $T=10.5, p=0.025$, respectively) and did not return to normal levels.

\section{DISCUSSION}

Numerous investigations suggest that HSP is not a self-limited disease as previously thought and may eventually develop into chronic kidney disease (CKD) in childhood [12]. The pathogenetic mechanism underlying HSP is poorly understood, but infectious agents and initiation of a diet have been suggested as triggers of an immune response [13]. Because HSP is one of the most well-known reasons for the development of CKD in children [14] it is important to find specific biomarkers of the development of renal syndrome in HSP patients. It was found that serum Gd-IgA1 and urinary $\operatorname{IgA}$, IgG, IgM, IL-6, IL-8, IL-10, and IgA-IgG and IgA-sCD89 complexes could identify HSP patients with renal involvement at the time of diagnosis. Elevated blood pressure and C3 level and decreased albumin are risk factors for renal damage in HSP patients [14].

HSP patients had significantly high levels of fibrinogen and leucocytes, which indicated the presence of an inflammation process, but these parameters were nonspecific. Platelets and fibrinogen have been reported to be important mediators of the activation of inflammation and coagulation $[3,15]$.

The results of standard coagulation assays show that prothrombin time and aPTT were within the normal ranges, even during the acute period, and did not differ significantly between the acute and remission periods. These results are concordant with those of previous studies $[3,16]$.

Among predisposing factors in the present study, the incidence of acute infectious disease was significantly higher. This is similar to trends seen in previous studies $[17,18]$.

Laboratory tests are not very useful in detecting HSP because even increased inflammatory markers such as CRP may be nonspecific [19].

Complement activation has been thought to play a role, but despite the demonstration of complement components in skin and renal biopsy material, serological evidence of complement activation is not convincing [20].

Our results confirm that activation of components C3 and $\mathrm{C} 4$ of the complement system is a feature of acute HSP. The similar nature of changes in the parameters of C3 and C4 fractions in HSP, as a representative of an immunocomplex disease, is a natural phenomenon, meaning that the complement system is involved in the formation and development of the autoimmune process and indicates consumption and depletion of the complement component as a result of the participation of these biolog- 
ical substances in inflammation, not compensated by the systems synthesising them.

In our study we showed that in patients with HSP, in the acute period of the disease, a serum decrease of $\mathrm{C} 3$ values in the HSPWN and HSPN groups was observed. A similar situation (decrease in C3 level) was recorded in both groups during the remission period of HSP. The C4 level was reduced in the group without involvement of renal syndrome during remission. The detected decreased levels of C3 and C4 in patients with HSP is consistent with the study of Lin et al. [21], who claimed that the association of hypocomplementaemia with HSP is a transient phenomenon. However, we did not observe this phenomenon because we explored the levels of serum of $\mathrm{C} 3$ and $\mathrm{C} 4$ after the disappearance of the main clinical features of HSP predominantly on the third week of the disease, which meant that the indicators did not return to normal levels.

The decrease of the C3 level of the component of the complement system was significantly reduced in the acute phase in cases with or without kidney involvement, which proved that hypocomplementaemia was not dependent on the severity of the process of HSP. This is in agreement with the study by Motoyama and Iitaka [22].

Changes in the parameters of coagulation haemostasis were recorded in children with renal involvement. We observed that the mean values of aPTT and prothrombin time were moderately reduced in both groups, which is evidence of activation of the coagulation potential of blood with an obvious tendency of the predominance of circulating blood system hypercoagulation. Such changes confirm the essential role of activation of system haemostasis in the mechanisms of development of HSP. It is clear that there is a relationship between the inflammation and coagulation systems. The activation of inflammation leads to the activation of the coagulation system, which also markedly affects inflammatory activity. This is considered important in the pathogeneses of vascular diseases $[23,24]$.

\section{CONCLUSIONS}

1. Abnormalities of the complement system in HSP have been reported. In the acute phase and remission of HSP a decreased value of C3 fraction was observed in serum in HSPWN and HSPN groups compared with the control group.

2. The decrease of the C3 level of components of the complement system was significantly reduced in the acute phase in cases with/without kidney involvement, which suggests that hypocomplementemia was not dependent on the severity of the process of HSP.

Study of the role of the membrane-activated complex in the enlargement and progression of HSP in children is planned.

\section{ACKNOWLEDGEMENTS}

The authors would like to thank the parents for allowing their children to participate in the study. We acknowledge the wholehearted support of the clinician's nurses and lab personnel who contributed and made this study possible.

This work was supported by the Kharkiv National Medical University (No. 0108U005255). The funder had no role in the study design, data collection and analysis, decision to publish, or preparation of the paper.

\section{DISCLOSURE}

The authors declare no conflict of interest.

\section{REFERENCES}

3. Kraft DM, Mckee D, Scott C. Henoch-Schönlein purpura: a review. Am Fam Physician 1998; 58: 405-408, 411.

4. Hung SP, Yang YH, Lin YT, et al. Clinical manifestations and outcomes of Henoch-Schönlein purpura: comparison between adults and children. Pediatr Neonatol 2009; 50: 162-168.

5. Hong J, Yang HR. Laboratory Markers Indicating Gastrointestinal Involvement of Henoch-Schönlein Purpura in Children. Pediatr Gastroenterol Hepatol Nutr 2015; 18: 39-47.

6. Pillebout E, Jamin A, Ayari H, et al. Biomarkers of IgA vasculitis nephritis in children. PLoS One 2017; 12: e0188718.

7. Sun L, Xie B, Zhang Q, et al. Biomarkers identification by a combined clinical and metabonomics analysis in Henoch-Schonlein purpura nephritis children. Oncotarget 2017; 8: 114239-114250.

8. Purevdorj N, Mu Y, Gu Y, et al. Clinical significance of the serum biomarker index detection in children with Henoch-Schonlein purpura. Clin Biochem 2018; 52: 167-170.

9. Welch TR. The complement system in renal diseases. Nephron 2001; 88: 199-204.

10. Yang YH, Tsai IJ, Chang CJ, et al. The interaction between circulating complement proteins and cutaneous microvascular endothelial cells in the development of childhood Henoch-Schönlein purpura. PloS One 2015; 10: e0120411.

11. Ozen S, Pistorio A, Iusan SM, et al. Criteria for Henoch-Schonlein purpura, childhood polyarteritisnodosa, childhood Wegener granulomatosis and childhood Takayasu arteritis: Ankara 2008. Part II: Final classification criteria. Ann Rheum Dis 2019; 69: 798-806.

10. https://assaypro.com/Products/Details/PC52122

11. https://assaypro.com/Products/Details/11221-05011

12. Davin JC, Coppo R. Henoch-Schönlein purpura nephritis in children. Nat Rev Nephrol 2014; 10: 563-573.

13. Saulsbury FT. Review Henoch-Schönlein purpura. Curr Opin Rheumatol 2010; 22: 598-602.

14. Chan H, Tang YL, Lv XH, et al. Risk factors associated with renal involvement in childhood Henoch-Schönlein Purpura: A MetaAnalysis. PLoS One 2016; 11: e0167346.

15. Szaba FM, Smiley ST. Roles for thrombin and fibrin(ogen) in cytokine/chemokine production and macrophage adhesion in vivo. Blood 2002; 99: 1053-1059.

16. Brendel-Müller K, Hahn A, Schneppenheim R, Santer R. Laboratory signs of activated coagulation are common in Henoch-Schönlein purpura. Pediatr Nephrol 2001; 16: 1084-1088. 
17. Jung DY, Kwon YR, Yu MH, et al. Clinical Features and Prognosis of Henoch-Schönlein Purpura in Children and Adults: A 13-Year Retrospective Study at a Single Centre. Child Kidney Dis 2017; 21: 61-68.

18. Saulsbury FT. Henoch-Schonlein Purpura in Children: Report of 100 Patients and Review of the Literature. Medicine 1999; 78: 395 409.

19. Yang HR. What We Know about Henoch-Schönlein Purpura in Children up to Date? Korean Med Sci 2018; 33: e199.

20. Smith GC, Davidson JE, Hughes DA, et al. Complement activation in Henoch-Schönlein purpura. Pediatr Nephrol 1997; 11: 477-480.

21. Lin Q, Min Y, Li Y, et al. Henoch-Schönlein purpura with hypocomplementemia. Pediatr Nephrol 2012; 27: 801-806.

22. Motoyama O, Iitaka K. Henoch-Schonlein purpura with hypocomplementemia in children. Pediatr Int 2005; 47: 39-42.

23. Levi M. Two-way interactions between inflammation and coagulation. Cardiovasc Med 2005; 15: 254-259.

24. Dolezalova P, Telekesova P, Nemkova D, Hoza J. Incidence of vasculitis in children in the Czech republic: 2-year prospective epidemiology survey. J Rheumatol 2004; 31: 2295-2299. 